\title{
Fiber optic muzzle brake tip for reducing fiber burnback and stone retropulsion during thulium fiber laser lithotripsy
}

Thomas C. Hutchens

David A. Gonzalez

Pierce B. Irby

Nathaniel M. Fried 


\title{
Fiber optic muzzle brake tip for reducing fiber burnback and stone retropulsion during thulium fiber laser lithotripsy
}

\author{
Thomas C. Hutchens, ${ }^{a}$ David A. Gonzalez, ${ }^{a}$ Pierce B. Irby, ${ }^{b}$ and Nathaniel M. Fried ${ }^{a, b, *}$ \\ anniversity of North Carolina at Charlotte, Department of Physics and Optical Science, 9201 University City Boulevard, Charlotte, \\ North Carolina 28223, United States \\ ${ }^{\mathrm{b}}$ Carolinas Medical Center, McKay Department of Urology, 1023 Edgehill Road South, Charlotte, North Carolina 28207, United States
}

\begin{abstract}
The experimental thulium fiber laser (TFL) is being explored as an alternative to the current clinical gold standard Holmium:YAG laser for lithotripsy. The near single-mode TFL beam allows coupling of higher power into smaller optical fibers than the multimode Holmium laser beam profile, without proximal fiber tip degradation. A smaller fiber is desirable because it provides more space in the ureteroscope working channel for increased saline irrigation rates and allows maximum ureteroscope deflection. However, distal fiber tip burnback increases as fiber diameter decreases. Previous studies utilizing hollow steel sheaths around recessed distal fiber tips reduced fiber burnback but increased stone retropulsion. A "fiber muzzle brake" was tested for reducing both fiber burnback and stone retropulsion by manipulating vapor bubble expansion. TFL lithotripsy studies were performed at $1908 \mathrm{~nm}, 35 \mathrm{~mJ}, 500 \mu \mathrm{s}$, and $300 \mathrm{~Hz}$ using a 100- $\mu \mathrm{m}$-core fiber. The optimal stainless steel muzzle brake tip tested consisted of a $1-\mathrm{cm}$-long, $560-\mu \mathrm{m}$-outer-diameter, 360- $\mu \mathrm{m}$-inner-diameter tube with a $275-\mu \mathrm{m}$-diameter through hole located $250 \mu \mathrm{m}$ from the distal end. The fiber tip was recessed a distance of $500 \mu \mathrm{m}$. Stone phantom retropulsion, fiber tip burnback, and calcium oxalate stone ablation studies were performed ex vivo. Small stones with a mass of $40 \pm 4 \mathrm{mg}$ and 4-mm-diameter were ablated over a 1.5-mm sieve in $25 \pm 4 \mathrm{~s}(n=10)$ without visible distal fiber tip burnback. Reduction in stone phantom retropulsion distance by $50 \%$ and $85 \%$ was observed when using muzzle brake tips versus $100-\mu \mathrm{m}$-core bare fibers and hollow steel tip fibers, respectively. The muzzle brake fiber tip simultaneously provided efficient stone ablation, reduced stone retropulsion, and minimal fiber degradation during TFL lithotripsy. ๑ 2017 Society of Photo-Optical Instrumentation Engineers (SPIE) [DOI: 10.1117/1.JBO.22.1.018001]
\end{abstract}

Keywords: ablation; burnback; fiber muzzle brake; lithotripsy; retropulsion; thulium; urinary stones.

Paper 160717PR received Oct. 17, 2016; accepted for publication Dec. 20, 2016; published online Jan. 10, 2017.

\section{Introduction}

\subsection{Holmium:YAG Laser Lithotripsy}

Approximately $10 \%$ of the U.S. population will suffer from urinary stone disease in their lifetime. In 2013, 49 million kidney stone cases occurred globally. ${ }^{1}$ To treat urinary stone disease, an endoscopic approach is commonly used with a laser fiber inserted through the working channel of a ureteroscope. This instrument is passed to the stone's location in the urinary tract where the stone is fragmented in place.

The Holmium:YAG laser is preferred by urologists because of its multiple uses in cutting or coagulating a variety of soft tissues and fragmentation of kidney stones, due to its relatively low cost, high power, and high water absorption in tissue at the emission wavelength of $2120 \mathrm{~nm}$. Limitations of this laser include a large multimode beam waist as well as limited capability in operating parameters such as pulse duration and pulse rate. Due to space limitations in the ureteroscope working channel, which typically has an inner diameter of $1.2 \mathrm{~mm}$, small optical fibers are desired to maximize other functions of the ureteroscope such as improved saline irrigation and maximal ureteroscope deflection, especially for accessing hard-to-reach

*Address all correspondence to: Nathaniel M. Fried, E-mail: nmfried@ uncc.edu locations in the lower pole of the kidney. However, suboptimal coupling of light into small core fibers with the Holmium laser's large multimode spatial beam profile risks overfilling of the input fiber core and launching excess light into the cladding layer, thus resulting in damage to the fiber on the proximal end, in addition to the typical damage on the distal end during stone fragmentation. Such damage may require the fiber to be repaired or replaced after each procedure. These limitations restrict coupling of Holmium laser energy into fibers with core diameters greater than $200 \mu \mathrm{m}$ in the clinic. $^{2,3}$

\subsection{Thulium Fiber Laser Lithotripsy}

The experimental thulium fiber laser (TFL) is being studied as an alternative to the current gold standard Holmium:YAG laser for lithotripsy. ${ }^{4-18}$ The TFL has a higher absorption coefficient $\left(\mu_{a} \sim 120 \mathrm{~cm}^{-1}\right)$ and shorter optical penetration depth in water, which translates into an ablation threshold four times lower compared with the Holmium laser $\left(\mu_{a} \sim 30 \mathrm{~cm}^{-1}\right)^{7,19,20}$ This property allows the TFL to ablate tissues at lower pulse energies than with the Holmium laser. Previous experiments have shown a wavelength-dependent correlation in ablation threshold to water absorption between Holmium and TFL. ${ }^{6,7}$ The TFL has 
two major emission wavelengths at 1908 and $1940 \mathrm{~nm}$, which closely match high and low temperature water absorption peaks, respectively. ${ }^{20-22}$ The diode-pumped TFL can also be electronically triggered to operate at variable pulse lengths of 100 to $1000 \mu \mathrm{s}$ and pulse rates of 1 to $1000 \mathrm{~Hz}$, compared to the Holmium laser, which is typically operated at pulse lengths of 350 to $700 \mu$ s and limited to pulse rates of 5 to $80 \mathrm{~Hz}$. These more flexible TFL parameters enable higher stone ablation rates than with the Holmium laser. ${ }^{10}$

A main advantage of the TFL is its Gaussian spatial beam profile. The superior spatial beam profile of a fiber laser improves coupling and transmission of laser power through small fibers for lithotripsy, allowing use of fiber core diameters less than $200 \mu \mathrm{m}^{4}$ and as low as $50 \mu \mathrm{m},{ }^{11}$ without proximal fiber tip damage. ${ }^{15}$ This reduction in fiber cross-section also enables increased ureteroscope deflection and saline irrigation rates through the working channel, ${ }^{5,23}$ which in turn may reduce procedure times, increase patient safety, and reduce probability of ureteroscope damage.

\subsection{Specialty Fiber Optic Tips}

A disadvantage of using smaller core fibers is the accelerated distal fiber tip degradation or burnback experienced during laser lithotripsy. ${ }^{5,24}$ Various methods have been studied to reduce distal fiber tip degradation, including tapered fiber tips, ${ }^{5}$ larger core detachable fiber tips, ${ }^{8}$ and ball tip fibers. ${ }^{14}$ All of these designs rely on the principle that larger diameter fibers degrade at a slower rate. Additionally, if the length of the larger distal fiber tip is sufficiently short (e.g., $<1 \mathrm{~cm}$ ), then it can be passed through the ureteroscope and still maintain the functional advantages of small core trunk fibers, such as increased ureteroscope deflection and saline irrigation rates. $5,11,13,25$

Fiber tip designs have also been previously employed to reduce distal fiber tip degradation and essentially eliminate fiber tip burnback simply by recessing the fiber tip a short distance inside a steel hypodermic tube. ${ }^{9}$ These studies showed that a greater recession depth would result in significant power loss due to the poor wave guiding ability of the hollow tip stemming from features on the tip surface larger than the TFL wavelength, while a shorter recession distance resulted in higher fiber tip degradation during stone ablation. A disadvantage of the hollow fiber tip was the increase in stone retropulsion force, believed to result from the confinement of the laser induced vapor bubble within the tube. The bubble expansion force was directed solely forward out of the tip of the tube, analogous to the barrel of a firearm, thus increasing retropulsion. A potential solution to this limitation is to integrate the fiber directly inside a stone basket, which would stabilize the stone and prevent retropulsion. ${ }^{13}$ However, this may not be a viable method for smaller stones, which may slip through the basket.

\subsection{Muzzle Brakes in Ballistics}

When a projectile is fired from the barrel of a weapon, the high pressure propellant gas following the bullet exits the muzzle or tip of the barrel with an axial force in the opposite direction, causing the barrel to recoil. Typically, the weapon's center of mass is off-axis, causing the barrel to rise after firing, and decreasing accuracy if the weapon is rapidly fired. A solution involves adding a muzzle brake or recoil compensator to the tip of the barrel. This allows a portion of the propellant gas to be directed laterally, thus reducing the recoil force axially.
A simple muzzle brake consists of porting the barrel near the muzzle with holes or slots.

The rapid vapor bubble expansion at the site of a submerged distal fiber tip during delivery of high laser pulse energy is somewhat analogous to the propellant gas exiting a weapon's barrel. The vapor bubble contributes to retropulsion of the stone during laser lithotripsy. ${ }^{9,16}$ By implementing a "fiber muzzle brake," the vapor bubble can be manipulated to potentially reduce stone retropulsion, analogous to the reduction of barrel recoil. This study introduces a low profile, distal fiber tip "muzzle brake" to reduce stone retropulsion for potential use in laser lithotripsy.

\section{Materials and Methods}

\subsection{Thulium Fiber Laser}

A 100-W, continuous-wave TFL (TLR 100-1908, IPG Photonics, Oxford, MA) with a center wavelength of $1908 \mathrm{~nm}$ was electronically modulated with a function generator (Model DS345, Stanford Research Systems, Sunnyvale, California) to produce a $35-\mathrm{mJ}$ pulse energy, 500- $\mu$ s pulse duration, and variable pulse rates up to $300 \mathrm{~Hz}$ for lithotripsy studies. The $500-\mu$ s pulse duration for the TFL was chosen to be within the range of the 350- to $700-\mu$ s pulse durations commonly used with the Holmium laser in the clinic. Longer TFL pulse durations (1 to $20 \mathrm{~ms}$ ) have been previously shown to produce increasing amounts of stone charring due to lack of thermal confinement ${ }^{6}$ that may lead to accelerated fiber tip degradation, while use of shorter pulse durations (e.g., $100 \mu \mathrm{s}$ ) does not provide sufficient energy per pulse for operation above the stone ablation threshold. A 25-mm-focal-length planoconvex lens (LA1560-D, Thorlabs, Newton, New Jersey) was used to focus the 5.5-mm-diameter $\left(1 / e^{2}\right)$ collimated fiber laser beam to a spot diameter of $25 \mu \mathrm{m}\left(1 / e^{2}\right)$ for coupling into the trunk fiber used in the study.

\subsection{Muzzle Brake Fiber Tip}

The low-OH, silica trunk fibers (FIPE100140170, Polymicro, Phoenix, Arizona) were cut to a length of $2 \mathrm{~m}$ and consisted of a $100-\mu \mathrm{m}$ core, $140-\mu \mathrm{m}$ outer diameter cladding, and $170-\mu \mathrm{m}$ outer diameter polyimide jacket. The protective jacket at the distal end of the trunk fiber was removed using a fiber stripper (Micro-Electronics, Seekonk, Massachusetts), exposing cladding for a $2 \mathrm{~mm}$ length. Stainless steel hypodermic tubing (B000FN1TVQ/B000FN3M98, Amazon Supply, Seattle, WA) with $180-\mu \mathrm{m}-\mathrm{ID}$ and $340-\mu \mathrm{m}-\mathrm{OD}$ was used as a "sheath" to mate and center the fiber to the $360-\mu \mathrm{m}-\mathrm{ID}$ and $560-\mu \mathrm{m}-\mathrm{OD}$ muzzle brake tube [Fig. 1(a)]. The sheath and muzzle brake tubing were cut to lengths of 10 and $5 \mathrm{~mm}$, respectively, to allow for independent manipulation during characterization studies. The portholes in the muzzle brake tip were drilled under magnification, using a stabilized high speed drill (Multi-Pro 395, Dremel, Racine, Wisconsin) and 275- $\mu$ m-diameter drill bits. A micromanipulator (460A-XYZ, Newport, Irvine, California) was used under magnification to recess the fiber and sheath in the muzzle brake tip to specified depths. Configurations were categorized by component distance to the distal end of muzzle brake $(\Delta z)$ and hole diameter $(\varnothing)$ [Fig. 1(b)]. For example, the configuration shown in Fig. 1(b) would be classified as follows: Hole $\varnothing-275-\mu \mathrm{m}$, Hole $\Delta z-500-\mu \mathrm{m}$, Fiber $\Delta z-600-\mu \mathrm{m}$, Sheath $\Delta z-750-\mu \mathrm{m}$. This terminology corresponds to a $275-\mu \mathrm{m}-$ diameter hole, centered at a distance of $500 \mu \mathrm{m}$ from the distal 
(a)
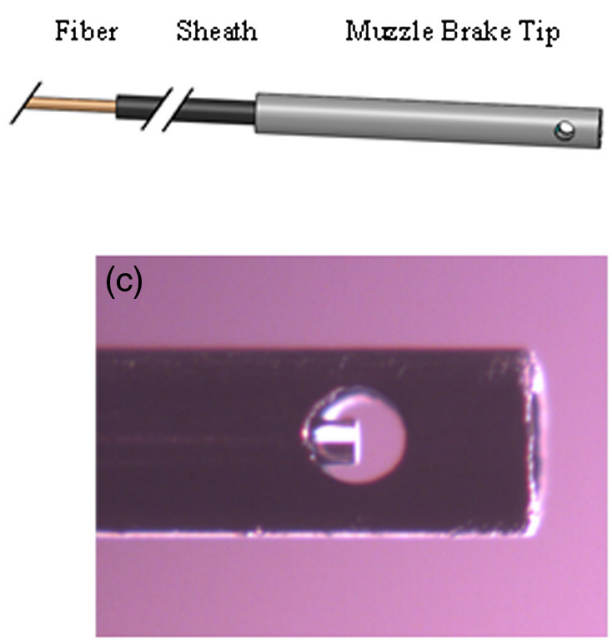
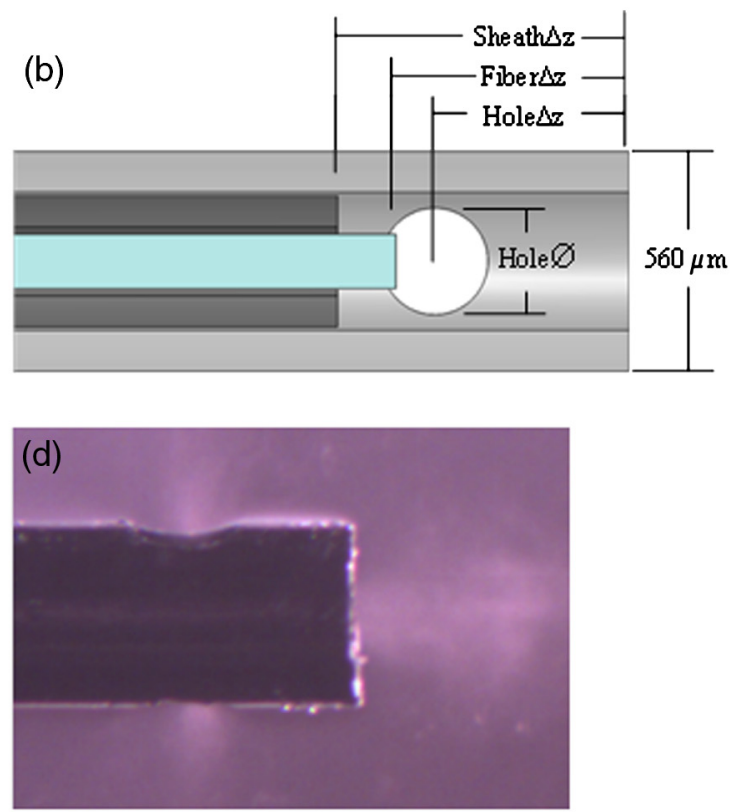

Fig. 1 (a) Scale representation of muzzle brake fiber tip constructed from stainless steel hypodermic tubing glued to the $170-\mu \mathrm{m}-\mathrm{OD}$ polyimide jacket of a trunk fiber. Muzzle brake tips tested were 5-mm-long and sheath 10-mm-long. (b) Cross-sectional model view of muzzle brake tip. Various configurations were tested and categorized by component distance to distal end of muzzle brake. The configuration shown would be classified as follows: Hole $\varnothing-275-\mu \mathrm{m}$, Hole $\Delta z-500-\mu \mathrm{m}$, Fiber $\Delta z-600-\mu \mathrm{m}$, Sheath $\Delta z-750-\mu \mathrm{m}$. This terminology corresponds to a $275-\mu \mathrm{m}$-diameter hole, centered at a distance of $500 \mu \mathrm{m}$ from the distal metal tip, with the bare fiber tip located $600 \mu \mathrm{m}$ from the distal metal tip, and the sheath located $750 \mu \mathrm{m}$ from the distal metal tip. (c) Photograph of a muzzle brake tip and (d) rotated 90 deg during laser operation.

metal tip, with the bare fiber tip located $600 \mu \mathrm{m}$ from the distal metal tip, and the sheath located $750 \mu \mathrm{m}$ from the distal metal tip. The fiber jacket, sheath, and brake tubes were bonded at set positions for lithotripsy studies using adhesive (Loctite 4203, Düsseldorf, Germany).

\subsection{Optical and Thermal Characterization}

Alignment, energy measurement, and optical transmission efficiency tests were performed by measuring the energy per pulse at a location before the trunk fiber and after the distal tip, before and after attachment of the muzzle brake tip, with a detector (PM150, Coherent, Santa Clara, California) connected to a meter (EPM2000, Molectron, Portland, Oregon). An insulated, 125 - $\mu \mathrm{m}$-diameter thermocouple (Type T, Omega, Norwalk, Connecticut) was used to monitor temperature as a function of time at 500-ms temporal resolution with a data acquisition system (OM-USB-TC, Omega), for the muzzle brake tip at various pulse rates during stone ablation. The thermocouple tip was glued in contact with the outer steel wall at a distance of $1 \mathrm{~mm}$ from the muzzle brake tip.

\subsection{Stone Retropulsion Setup}

Laser energy was delivered through muzzle brake tip fibers and bare fibers in contact with 6-mm-diameter spherical stone phantoms, submerged in water. Commercial Airsoft pellets (SAP5020, Crosman Corporation, Bloomfield, New York) were chosen for their consistent size, mass $(206.7 \pm 2.8 \mathrm{mg})$, and low cost. While these plastic pellets may not provide a reliable phantom for ablation studies, the primary contributor to retropulsion is vapor bubble expansion, not ejection of stone particles.

Two experimental setups were used to characterize stone retropulsion. First, a custom $\mathrm{V}$-shaped trough with a ruler was constructed and leveled horizontally [Fig. 2(a)]. A V-shape was chosen so that ablation debris from the phantoms could settle without affecting the sliding or rolling of the spheres. Cameras with magnification were used to properly position the fiber tip to the phantom's equator in contact and the fiber tip to zero on the trough's ruler. Measurements were recorded based on stone distance traveled in the trough. A total energy of $42 \mathrm{~J}$ was delivered to each stone phantom, translating into an operating time of 4, 6, and $12 \mathrm{~s}$ for 300,200 , and $100 \mathrm{~Hz}$, respectively. A minimum of 10 stone retropulsion trials were completed for each muzzle brake tip configuration.

Second, a pendulum setup was utilized to characterize retropulsion [Fig. 2(b)], since the trough setup may lose sensitivity with small retropulsion forces due to static friction. A stone phantom was suspended by 1-kg-test fishing line measuring $0.200 \mathrm{~m}$ from the pivot point to the center of the phantom. While the pendulum was only partially submerged, air turbulence on the line did not appear to be a concern due to the vibrational damping of the water. Custom clear side walls with a 7.5-mm gap were used to confine the pendulum motion to one direction with a 5-mm-grid ruler on the back wall. The muzzle brake tips were oriented so that the portholes faced vertically, not into the side walls, allowing for isolation of the forward retropulsion force. Cameras with magnification were used to properly position the fiber tip to the phantom's equator $1 \mathrm{~mm}$ away from the stone phantom at equilibrium. In the trough setup, the majority of the momentum was delivered to the stone phantom 

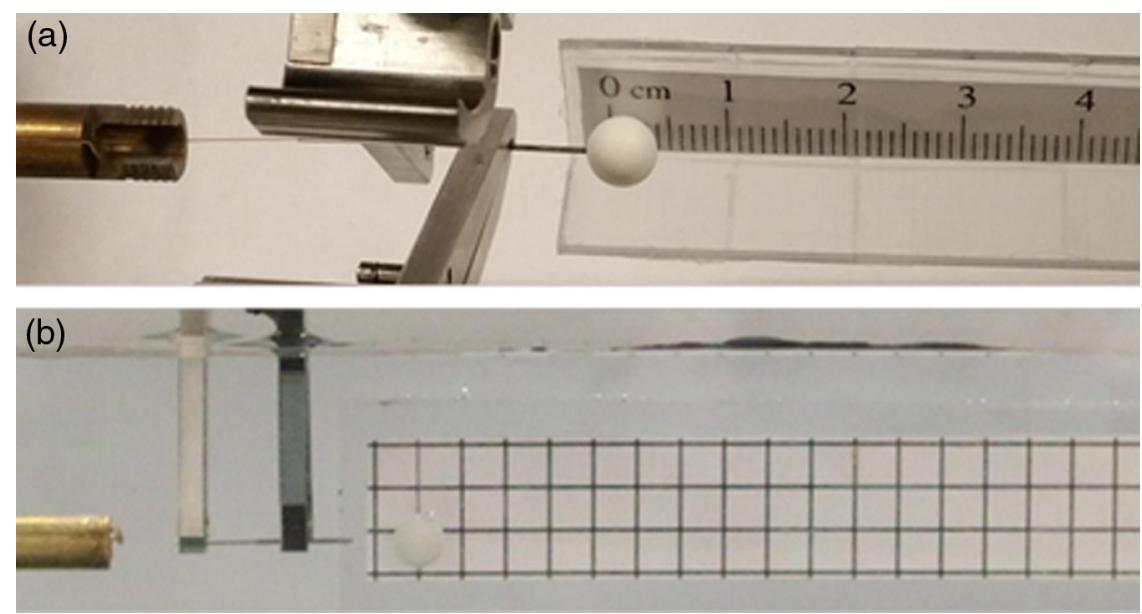

Fig. 2 Stone retropulsion setups with independent muzzle brake, sheath and optical fiber manipulation. (a) Top view of trough setup where stone phantom rolls/slides in a V-shaped channel. (b) Side view of pendulum setup where stone phantom is suspended and swings from equilibrium with a grid ruler marked in $5 \mathrm{~mm}$ increments.

with the initial pulse, and this could cause the stone to roll to great distances, sometimes independent of laser operating time. With the pendulum setup, the stone displacement from equilibrium was measured in steady state during laser operation, at $0.5 \mathrm{~mm}$ resolution, by video.

\subsection{Thulium Fiber Laser Lithotripsy Setup}

Small kidney stone samples composed of $60 \%$ calcium oxalate monohydrate and $40 \%$ calcium phosphate $(\mathrm{COM} / \mathrm{CP})$ were obtained from a single source and had consistent mass ( 40 mg), size (4 mm), shape, and color. Also, large ( $8 \mathrm{~mm}$, $150 \mathrm{mg}$ ) COM urinary stone samples with $>95 \%$ purity were obtained from a stone analysis laboratory (LabCorp, Oklahoma City, Oklahoma). These stones were chosen because calcium oxalate stones comprise about $80 \%$ of all stone compositions encountered in the clinic. ${ }^{26}$ Stone samples were desiccated in an oven at $70^{\circ} \mathrm{C}$ for $15 \mathrm{~min}$ and then weighed with an analytical balance (Model AB54-S, Mettler-Toledo, Columbus, Ohio) before experiments to determine initial mass. The samples were then placed on a $1.5-\mathrm{mm}$ wire mesh sieve and immersed in saline (Fig. 3), similar to previously reported experiments. ${ }^{14}$ TFL lithotripsy experiments were conducted after a rehydration time of $1 \mathrm{~min}$, using a muzzle brake fiber or bare fiber held by

(a)

(b)

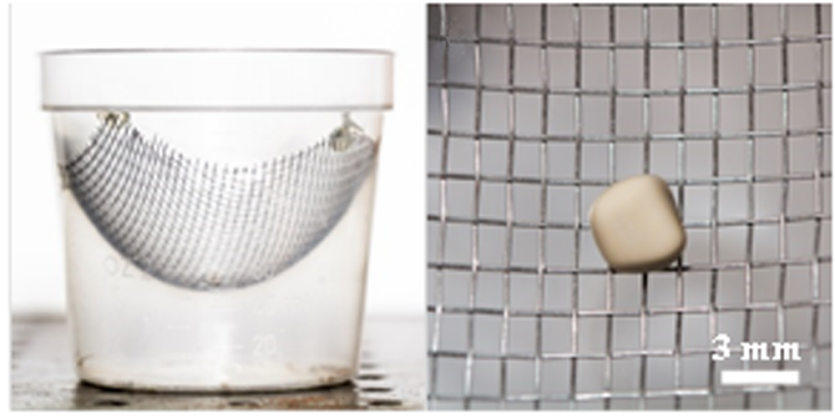

Fig. 3 (a) 1.5-mm mesh sieve integrated into transparent plastic container filled with saline. (b) $60 \%$ calcium oxalate monohydrate $/ 40 \%$ calcium phosphate kidney stone resting on mesh sieve. hand with a fiber chuck. Stone ablation operation time was recorded for small stones and the ablation rate was calculated by dividing the stone's change in mass by laser operation time for larger stones.

\subsection{Fiber Tip Degradation}

Distal fiber tip damage inspection was conducted using a digital camera (EOS 70D, Canon, Tokyo, Japan) coupled to a fiber optic microscope (FS200, Thorlabs) to capture images before and after stone ablation. The proximal fiber tip was illuminated with white light (MI-150, Dolan-Jenner, Boxborough, Massachusetts) so distal fiber tip degradation could be observed through the microscope eyepiece.

\section{Results}

\subsection{Optical and Thermal Measurements}

Bare fiber power output measured $10.2 \pm 0.2 \mathrm{~W}$ at a pulse rate of $300 \mathrm{~Hz}$, corresponding to an energy per pulse of $34 \pm 1 \mathrm{~mJ}$. After the muzzle brake tip was attached, this same power output was confirmed if the fiber was recessed less than $500 \mu \mathrm{m}$ and the sheath was recessed beyond the fiber. This was due to a significant portion of light diverging out of the fiber and hitting the steel before escaping the tip. Energy measurements were also recorded after the stone ablation studies to check functionality. If the fiber tip degraded, the measured energy would typically drop to less than $25 \mathrm{~mJ}$. The TFL ablation threshold has been reported to be $20 \mathrm{~J} / \mathrm{cm}^{2}$ for COM stones. ${ }^{7}$ Assuming the degraded tip output would be confined over the $360-\mu \mathrm{m}$ inner diameter of the muzzle brake tip, this would equate to an energy density of less than $6 \mathrm{~J} / \mathrm{cm}^{2}$. For comparison, a nondegraded $100-\mu \mathrm{m}$-core fiber emitting $35 \mathrm{~mJ}, 500 \mu \mathrm{m}$ from steel tip $\left(\mathrm{NA}=0.22\right.$ in air) in saline (spot size $\left.=0.056 \mathrm{~mm}^{2}\right)$ has an energy density of $60 \mathrm{~J} / \mathrm{cm}^{2}$.

Tests utilizing a thermocouple attached to the muzzle brake tip [Hole $\varnothing-275-\mu \mathrm{m}$, Hole $\Delta z-250-\mu \mathrm{m}$, THRU, Fiber $\Delta z-500-\mu \mathrm{m}$, Sheath $\Delta z-1000-\mu \mathrm{m} ;$ Fig. 4(a)] showed that when laser operation occurred within a saline bath, the temperature on the outside of the steel tip increased less than $10^{\circ} \mathrm{C}$ at a pulse rate of $300 \mathrm{~Hz}$ for about $30 \mathrm{~s}$. When a small COM/CP stone $(\sim 40 \mathrm{mg})$ was ablated 
(a)
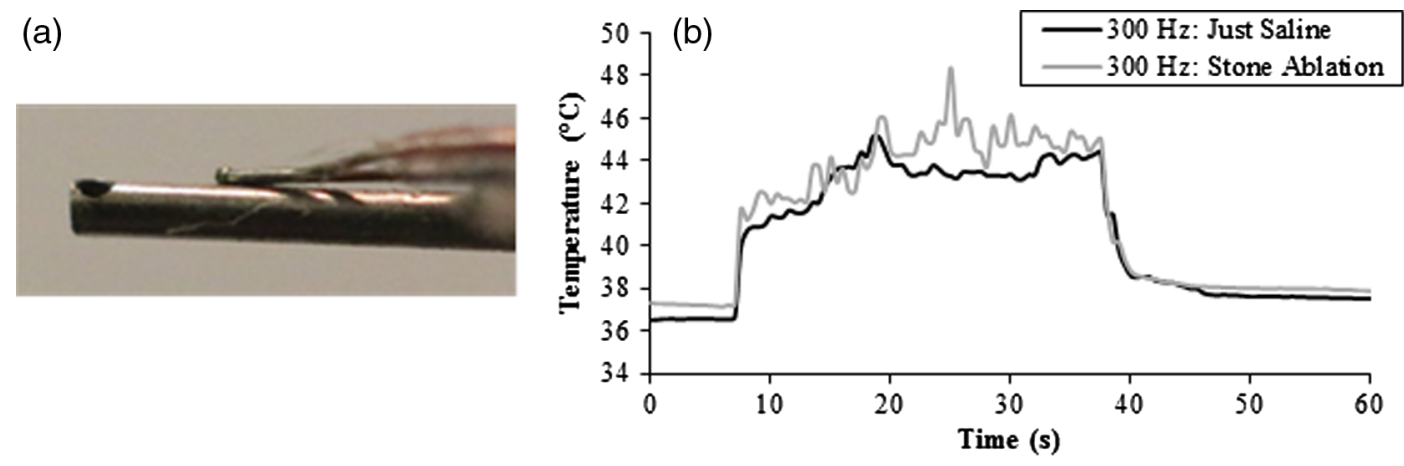

Fig. 4 (a) Image of thermocouple placed $1 \mathrm{~mm}$ from the muzzle brake tip; (b) thermocouple plots showing fiber muzzle brake outer steel temperature at $300 \mathrm{~Hz}$ in saline and during COM/CP stone ablation. Studies were performed in a saline bath heated to body temperature $\left(37^{\circ} \mathrm{C}\right)$.

through a sieve at $300 \mathrm{~Hz}$, the temperature fluctuated, but remained below $50^{\circ} \mathrm{C}$, demonstrating a favorable safety profile for clinical studies [Fig. 4(b)]. Active saline irrigation during clinical procedures would result in even lower saline temperatures, further improving the safety profile.

\subsection{Stone Phantom Retropulsion (Trough Setup)}

Figure 5 shows retropulsion distances $(\mathrm{mm})$ as a function of fiber recession distance $(\mu \mathrm{m})$ for the trough setup. When the muzzle tip (Hole $\varnothing-275-\mu \mathrm{m}$, Hole $\Delta z-500-\mu \mathrm{m}$, THRU, Sheath $\Delta z-600-\mu \mathrm{m})$ portholes were covered by the sheath, an increase in retropulsion distance was observed, similar to previous hollow tip studies. ${ }^{9}$ Between a recession distance of 250 and $600 \mu \mathrm{m}$, the muzzle tip reduced stone retropulsion below the baseline retropulsion level of $5.8 \pm 2.4 \mathrm{~mm}$ for the bare $100-\mu \mathrm{m}$ fiber.

\subsection{Stone Phantom Retropulsion (Pendulum Setup)}

The pendulum retropulsion setup was first used to characterize a bare fiber operating at different pulse rates (Fig. 6), with fixed pulse energy of 35-mJ and 500- $\mu$ s pulse duration. Pulse rates up to $500 \mathrm{~Hz}$ are shown, however, previous reports have demonstrated that pulse rates higher than $300 \mathrm{~Hz}$ can reduce fiber lifetime by generating additional heat in the saline medium, without

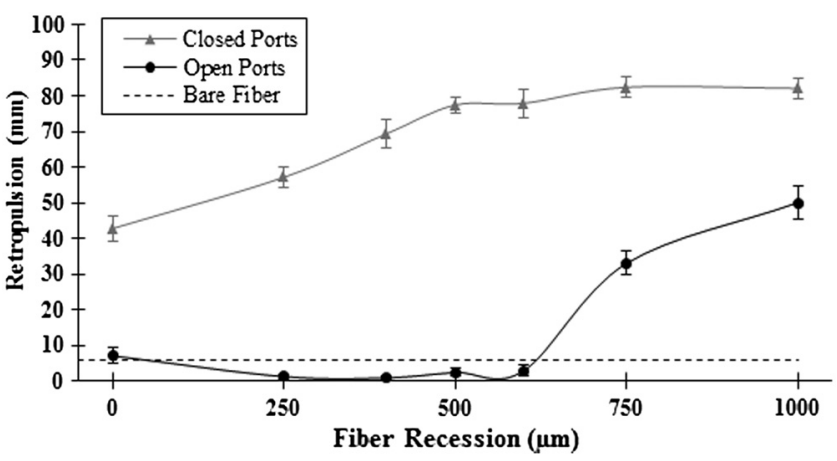

Fig. 5 Trough setup stone phantom retropulsion distance versus fiber recession distance from tip for hollow tip (gray), and muzzle brake tip (black) with hole and sheath at 500 and $600 \mu \mathrm{m}$, respectively. Bare fiber retropulsion in contact (working distance $=0$ ) is shown with dashed line for reference $(5.8 \pm 2.4 \mathrm{~mm})$. All measurements were performed with similar laser settings ( $35 \mathrm{~mJ}, 500 \mu \mathrm{s}, 300 \mathrm{~Hz})$ and total energy of $42 \mathrm{~J}(N=10)$. increasing stone ablation rates. ${ }^{10} \mathrm{~A}$ nearly linear correlation between pulse rate and stone retropulsion is observed at pulse rates up to $500 \mathrm{~Hz}$, however, the pendulum restoration force will become nonlinear as the displacement increases significantly. A pulse rate of $300 \mathrm{~Hz}$ was used for the remainder of these studies, corresponding to a pendulum displacement or retropulsion distance of $5.5 \mathrm{~mm}$ for a bare fiber.

To better understand how the porthole area affects stone retropulsion, the size and quantity of holes were varied, while hole position remained $1 \mathrm{~mm}$ from the steel tip. This large depth was chosen to exclude effects close to the distal tip and isolate what occurs when the fiber is merely near the portholes. Three hole sizes were studied up to $350 \mu \mathrm{m}$, corresponding to the tube's inner diameter. Since a $350 \mu \mathrm{m}$ "CROSS" (4) hole structure at the same position would sever the tip of the steel tube, $275 \mu \mathrm{m}$ diameter hole configurations were used for characterization of hole quantity. The sheath position remained constant at $1500 \mu \mathrm{m}$. An undrilled outer tube was also measured for comparison. All configurations are shown in Fig. 7 as well as a visualization of position $(1 \mathrm{~mm})$ of a median hole size $(275 \mu \mathrm{m})$. Use of a single hole produced a decrease in retropulsion compared to the closed tube, and multiple holes showed a decrease when compared to a single hole. However, no difference was observed for a $275 \mu \mathrm{m}$ THRU (2) versus a CROSS (4). The $275 \mu \mathrm{m}$ THRU hole was chosen for further testing since it produced a decrease in retropulsion while maintaining the structural integrity of the steel tube.

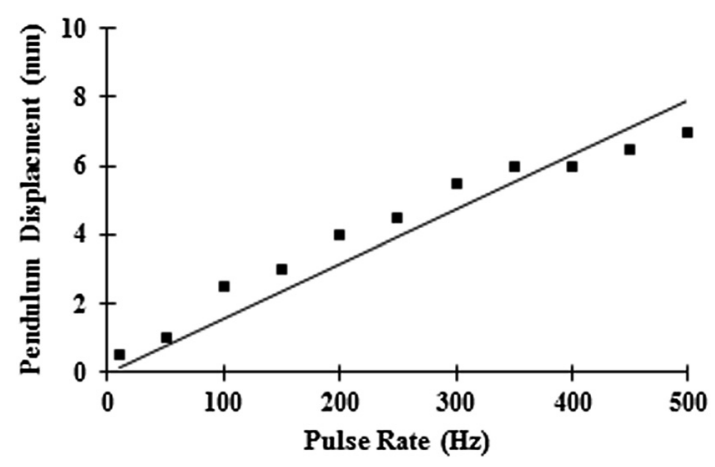

Fig. 6 Stone phantom retropulsion as a function of pulse rate $(35 \mathrm{~mJ}$, $500 \mu \mathrm{s})$ for a bare fiber $(100-\mu \mathrm{m}$-core, $140-\mu \mathrm{m}$-cladding) using a 200-mm-length, 200-mg-mass pendulum. Studies were conducted at $300 \mathrm{~Hz}$, corresponding to a bare fiber pendulum displacement of $\sim 5.5 \mathrm{~mm}$. A linear fit is shown that should theoretically be valid up to $1000 \mathrm{~Hz}$, or $50 \%$ duty cycle, for a pulse duration of $500 \mu \mathrm{s}$. 


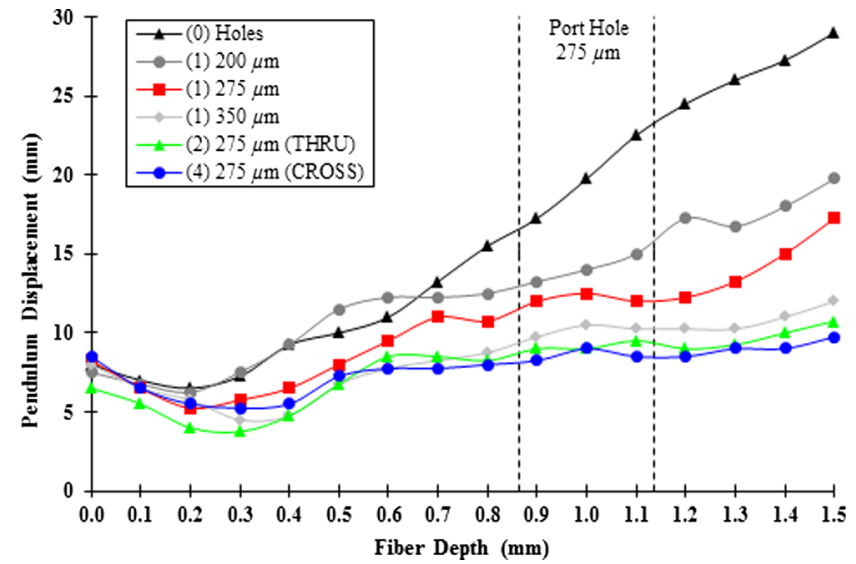

Fig. 7 Stone phantom retropulsion as a function of size and quantity of holes (35 mJ, $500 \mu \mathrm{s}, 300 \mathrm{~Hz}$ ) using a 200-mm-length, 200-mgmass pendulum. All holes are centered $1 \mathrm{~mm}$ from the tip of the muzzle brake (between dashed vertical lines), and the sheath is fixed at a position of $1.5 \mathrm{~mm}$.
Retropulsion for muzzle brake tips (Hole $\varnothing-275-\mu \mathrm{m}$, THRU) was fully characterized by recessing the fiber and sheath by 100 and $200 \mu \mathrm{m}$ increments, respectively, with hole positions centered at 250 and $500 \mu \mathrm{m}$, as shown in Figs. 8 and 9, respectively. In some configurations, the sheath was placed in front of the portholes, effectively closing them and imitating a hollow tip instead of a muzzle brake. These configurations were measured to better understand the dynamics of the muzzle brake. When the sheath was positioned behind the portholes of the outer tube in each case, retropulsion was reduced, since the portholes were open. As the fiber was recessed beyond the portholes, there was a slight dip then a steady increase in retropulsion. The lowest observed retropulsion with the muzzle brake $(2.5 \mathrm{~mm})$ was half that observed with the bare fiber $(5.5 \mathrm{~mm})$. While all sheath positions beyond the portholes showed no change in retropulsion, a position of $1000 \mu \mathrm{m}$ was chosen for lithotripsy studies to potentially keep the fiber tip cooler through the presence of an increased amount of saline. Recessing the sheath too far could potentially decenter the fiber and increase fiber vibration within the muzzle brake.

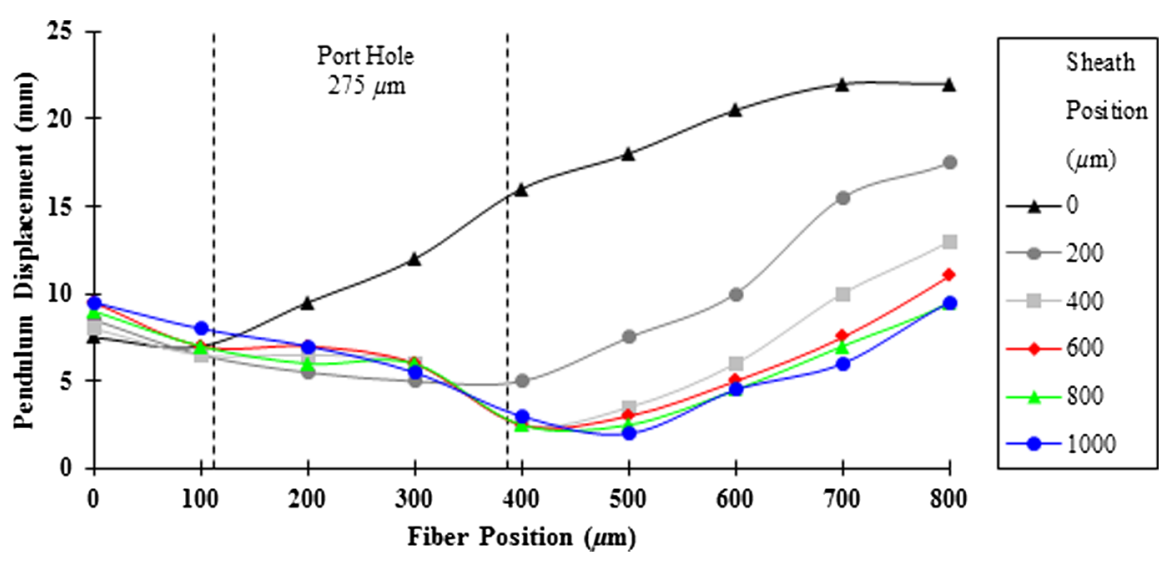

Fig. 8 Muzzle brake fiber tip (Hole $\varnothing-275-\mu \mathrm{m}$, Hole $\Delta z-250-\mu \mathrm{m}$, THRU) retropulsion as a function of fiber and sheath position using a $200-\mathrm{mm}$-length, 200 -mg-mass pendulum. Each line corresponds to a different sheath recession distance $(\mu \mathrm{m})$ from the tip. The dashed vertical lines illustrate the location and size of the portholes.

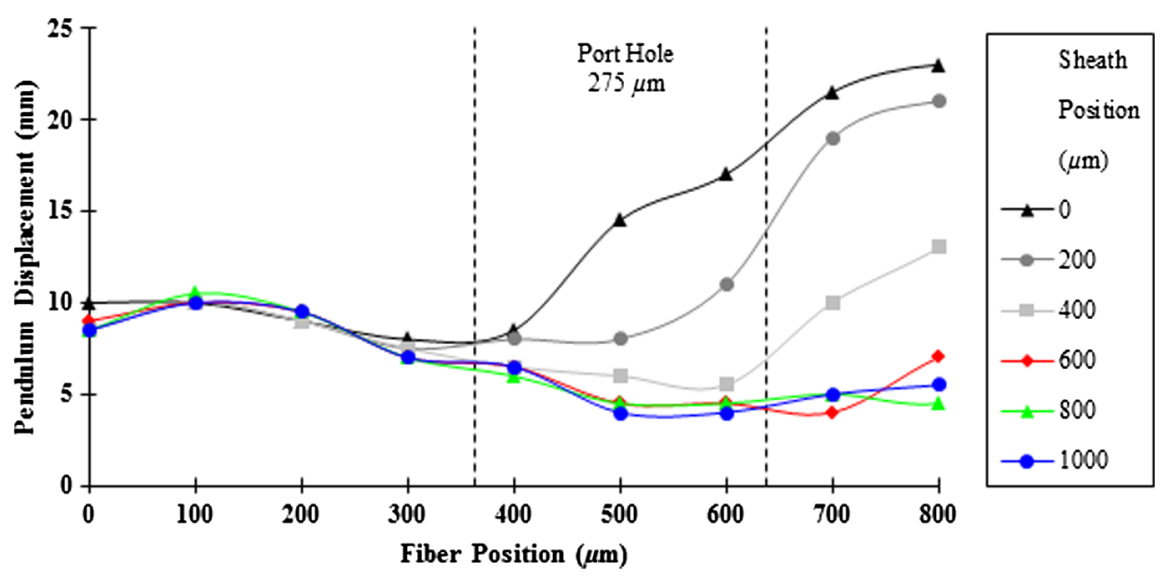

Fig. 9 Muzzle brake fiber tip (Hole $\varnothing-275-\mu \mathrm{m}$, Hole $\Delta z-500-\mu \mathrm{m}$, THRU) retropulsion as a function of fiber and sheath position using a 200-mm-length, 200-mg-mass pendulum. Each line corresponds to a different sheath recession distance $(\mu \mathrm{m})$ from the tip. The dashed vertical lines illustrate the location and size of the portholes. 


\subsection{Thulium Fiber Laser Lithotripsy}

Table 1 shows data obtained when the muzzle brake fiber tip and bare fiber tip were used to ablate 4-mm-diameter COM/CP stone samples until all fragments were sufficiently small enough ( $<1.5 \mathrm{~mm}$ diameter) to pass through the sieve. The muzzle brake tip operating time was $25 \%$ slower than the bare fiber operating time. However, no fiber tip degradation was observed for the muzzle brake after all $10 \mathrm{COM} / \mathrm{CP}$ stones, compared to the bare fiber, which burned back steadily over the entire operation time.

Table 1 Stone ablation operation times for $4 \pm 1 \mathrm{~mm}$ diameter $\mathrm{COM} /$ $\mathrm{CP}$ stone samples over a $1.5-\mathrm{mm}$ sieve. Bare fiber $(100-\mu \mathrm{m}$-core, $140-\mu \mathrm{m}$-cladding) was compared to muzzle brake tip (Hole $\varnothing-275-\mu \mathrm{m}$, Hole $\Delta z-250-\mu \mathrm{m}$, Fiber $\Delta z-500-\mu \mathrm{m}$, Sheath $\Delta z-1000-\mu \mathrm{m})$. Distal fiber tip burnback rate after all 10 samples is also shown ( $35 \mathrm{~mJ}, 500 \mu \mathrm{s}$, $300 \mathrm{~Hz})$.

\begin{tabular}{lccccc} 
& \multicolumn{2}{c}{ Muzzle brake tip } & & \multicolumn{2}{c}{ Bare Fiber } \\
\cline { 2 - 3 } \cline { 5 - 6 } Sample \# & Mass $(\mathrm{mg})$ & Time $(\mathrm{s})$ & & Mass $(\mathrm{mg})$ & Time $(\mathrm{s})$ \\
\hline 1 & 31.4 & 20 & & 34.9 & 15 \\
2 & 39.3 & 25 & & 34.7 & 18 \\
3 & 45.0 & 28 & & 46.0 & 24 \\
4 & 45.3 & 33 & & 35.6 & 13 \\
5 & 39.4 & 26 & & 36.2 & 14 \\
6 & 40.9 & 25 & & 41.5 & 23 \\
7 & 41.0 & 22 & & 41.8 & 19 \\
8 & 42.3 & 26 & & 43.1 & 24 \\
9 & 39.2 & 29 & & 41.7 & 24 \\
10 & 38.3 & 18 & & 41.8 & 25 \\
Mean \pm Stdev* & $40 \pm 4$ & $25 \pm 4$ & $40 \pm 4$ & $20 \pm 5$ \\
Fiber burnback & None & & $570 \mu \mathrm{m} / \mathrm{min}$ & \\
\hline
\end{tabular}

*Statistical analysis: mean mass is similar $(p=0.39)$; mean time is significant $(p=0.01)$.
Large COM stones (8-mm-diameter, $150 \mathrm{mg}$ ) were also tested to provide a more robust calculation of ablation rate by means of mass loss over longer operating times. The bare fiber produced an ablation rate of $0.43 \mathrm{mg} / \mathrm{s}$. Bare fiber tip burnback rate was also observed to decrease with smaller stone size, probably due to less probability of the fiber tip being lodged in crevices on the stone surface and overheating. At the beginning of the ablation procedure (stone size of $8 \mathrm{~mm}$ ), burnback was over $3000 \mu \mathrm{m} / \mathrm{min}$. Once the stone was smaller ( $<5 \mathrm{~mm}$ ), burnback decreased to about $1000 \mu \mathrm{m} / \mathrm{min}$.

\subsection{Fiber Tip Degradation}

Images taken of fiber tip degradation before stone ablation showed a relatively smooth fiber tip surface [Fig. 10(a)]. After $n=10$ stones were ablated, the fiber tip was imaged again [Fig. 10(b)]. Fiber burnback was qualitatively studied, with no visible burnback appearing after treatment of 10 stones.

\section{Discussion}

\subsection{Thulium Fiber Laser Advantages}

Our laboratory is studying the experimental TFL as a potential alternative to the current gold standard Holmium:YAG laser for lithotripsy. TFL technology offers several advantages compared to the conventional Holmium laser. First, the TFL wavelength of $1908 \mathrm{~nm}$ more closely matches a high-temperature water absorption peak in tissue at $1910 \mathrm{~nm}$, leading to a four times lower TFL stone ablation threshold than for the Holmium laser wavelength at $2120 \mathrm{~nm}^{7}$ Second, the TFL near-single mode spatial beam profile allows delivery of high power $(>10 \mathrm{~W})$ through smaller fibers (e.g., 50 or $100 \mu \mathrm{m}$ core) than currently used with the multimode Holmium laser (>200 $\mu \mathrm{m}$ core). ${ }^{4,5,11}$ Third, use of smaller fibers enables higher irrigation rates through the single working channel of the ureteroscope for increased visibility and safety. ${ }^{5,11}$ Fourth, use of smaller fibers also enables greater ureteroscope deflection, which should translate into a lower probability of the fiber breaking inside the ureteroscope, which is expensive to repair. ${ }^{5}$

\subsection{Muzzle Brake Fiber Tip Design and Safety Tests}

Multiple muzzle brake tip configurations were tested, balancing minimal fiber tip degradation, minimal stone retropulsion, and

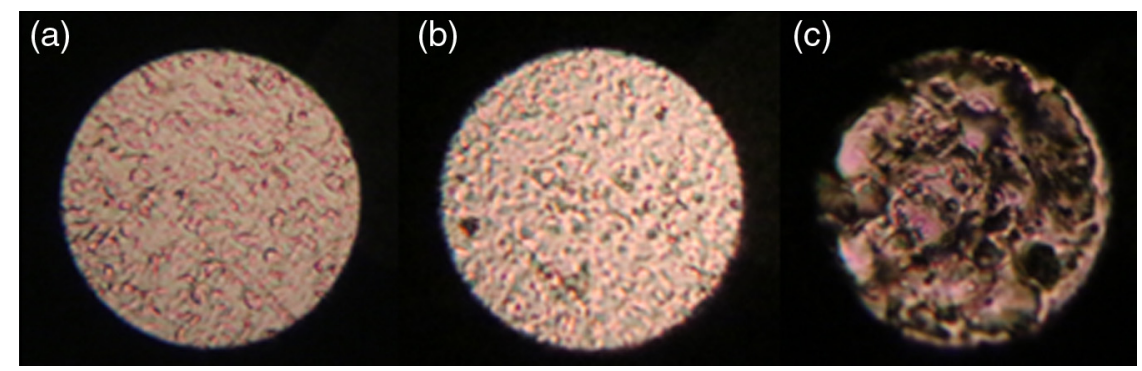

Fig. 10 (a) $100-\mu \mathrm{m}$-core distal fiber tip before ablation, polished with $16-\mu \mathrm{m}$-grit pad. (b) Fiber tip of muzzle brake (Hole $\varnothing-275-\mu \mathrm{m}$, Hole $\Delta z-250-\mu \mathrm{m}$, Fiber $\Delta z-500-\mu \mathrm{m}$, Sheath $\Delta z-1000-\mu \mathrm{m}$ ), after ablation of 10 small COM/CP stones totaling, $\sim 75,000$ laser pulses. (c) Fiber tip degradation caused by overheating, either by stone debris in close proximity during laser pulse and/or poor irrigation of muzzle brake, which can occur when "drilling" into a stone instead of surface ablation. 
sufficient stone ablation rates, yielding an optimal configuration where the fiber was recessed between 250 and $500 \mu \mathrm{m}$ into the muzzle tip. A distance less than $250 \mu \mathrm{m}$ produced a large increase in fiber degradation, possibly due to increased debris and heating from stone ablation.

Thermal tests were also conducted to confirm safe operation for potential lithotripsy procedures in the clinic. Without a stone present, saline temperatures increased from $37^{\circ} \mathrm{C}$ to $45^{\circ} \mathrm{C}$. During COM/CP stone ablation, temperatures peaked at $48^{\circ} \mathrm{C}$, which is judged safe for the clinic $\left(<60^{\circ} \mathrm{C}\right)$. A worst-case scenario failure test was also performed by purposely "drilling" the muzzle brake tip into a large COM stone, resulting in fiber tip degradation from overheating. In this case, a maximum temperature of $54^{\circ} \mathrm{C}$ was recorded, lasting less than $1 \mathrm{~s}$. Active cooling with room temperature saline irrigation, currently performed during Holmium laser lithotripsy in the clinic, should lower the peak temperatures even further, thus improving the safety profile. The vapor exiting the side holes may cause a temperature rise at the urothelium, however, the duty cycle (on/off time) is only 1:7 for the maximum pulse rate of $300 \mathrm{~Hz}$. Also, unlike the forward expanding vapor bubble, no laser energy is exiting from the side holes. Tissue heating by the progressive heating of the steel tip itself may be more significant than the vapor exiting the side holes, although this requires further study.

Specialty fiber tips with larger diameters than the trunk fiber have been previously studied with ureteroscope deflection and saline irrigation rates tested. ${ }^{5}$ The 10 -mm length of the muzzle brake tip was chosen for ease of manipulation during characterization studies and could potentially be made shorter and/or smaller in diameter for clinical application to maintain maximum ureteroscope deflection. While larger core trunk fibers ( $>200-\mu \mathrm{m}-\mathrm{OD}$ ) have been shown to reduce ureteroscope deflection, smaller core trunk fibers $(<200-\mu \mathrm{m}-\mathrm{OD})$ with shorter length $(<10 \mathrm{~mm})$ and larger diameter specialty tips near the distal end of the ureteroscope do not impede ureteroscope deflection. ${ }^{5}$ The fiber tip may remain nestled within the ureteroscope's typical semirigid distal tip section during ureteroscope navigation. Then the fiber may be extended from the ( 1.2-mm-ID) working channel during lithotripsy procedures to improve irrigation rates, which are dependent on the working channel cross-sectional area.

\subsection{Stone Retropulsion}

Reduction in stone phantom retropulsion distance by $50 \%$ and $85 \%$ was observed when using muzzle brake tips versus $100-\mu \mathrm{m}$-core bare fibers and hollow steel tip fibers, respectively, but at the expense of lower ablation rates. The pendulum setup provided faster and more repeatable results compared to the trough setup. The friction and leveling uncertainty of the trough showed higher variability between studies. To calculate actual forward force from the fiber or muzzle brake using the pendulum, other factors would need to be considered, including the pendulum restoration force and change in the stone position from the fiber tip, however, only the stone position was reported in this study. The data were collected from the pendulum in steady state, therefore, the tendency should be consistent between muzzle brakes with the same configuration and with only slight variations in magnitude based on tip construction quality. An alternative pendulum method was considered involving translating the pivot point of the pendulum until the stone phantom was in contact with the muzzle brake tip, then measuring the angle from equilibrium to provide a direct force calculation. However, laser ablation of the phantom could not be decoupled from the force of the vapor bubble in this method, and the stone mass would be inconsistent, thus hindering steady state measurements.

Initially, minimum retropulsion was estimated to be achieved when the fiber was recessed from the muzzle brake tip's portholes, with the metal sheath $100 \mu \mathrm{m}$ behind the fiber tip. After testing, the data showed that if the fiber recession was less than the sheath recession depth, and the muzzle holes were open, then stone phantom retropulsion would decrease below baseline retropulsion for a bare fiber in contact with a stone. When the muzzle brake tip fiber was in contact with a stone phantom, similar retropulsion distances were observed as compared to a bare fiber. Tip configurations that reduced retropulsion below baseline for a bare fiber were chosen for ablation studies. When fiber recession distance was zero and sheath recession depth was also zero, retropulsion was observed to increase in comparison to a bare fiber. In this specific case, the metal tip simulated a "launching pad," with 560- $\mu$ m-diameter, and thus behaved similarly to a large core fiber. Large core fibers have been reported to produce greater stone retropulsion when used with the Holmium laser. ${ }^{27-30}$ The basic muzzle brake design could also be used to reduce stone retropulsion with the Holmium laser, however, the dimensions of the muzzle brake would need to be larger to accommodate the larger trunk fibers typically used with the Holmium laser.

As an aside, it should be noted that recent studies have also reported a reduction in stone retropulsion for the Thulium:YAG laser in comparison to the Holmium:YAG laser, using standard 270- $\mu$ m optical fibers. ${ }^{31}$ However, the solid-state Thulium:YAG laser should not be confused with the TFL used in our study, since their laser parameters differ, and therefore, comparisons between the two different types of thulium lasers cannot be easily inferred.

\subsection{Thulium Fiber Laser Lithotripsy}

TFL stone ablation rates for the muzzle brake tips $(40 \pm 4 \mathrm{mg}$ over $25 \pm 4 \mathrm{~s}$ ) when the fiber and sheath were recessed 500 and $1000 \mu \mathrm{m}$ into the muzzle tip, respectively, were comparable to previously reported data conducted with similar stone type and laser parameters $(35 \mathrm{~mJ}, 500 \mu \mathrm{s}$, and $300 \mathrm{~Hz})$ and a bare fiber tip (71 $\pm 6 \mathrm{mg}$ over $54 \pm 9 \mathrm{~s}) .{ }^{14}$ Slightly larger stones (4 to $5 \mathrm{~mm}$ ) were used in these previous studies, which led to longer operation times. ${ }^{14}$ Bare fiber ablation studies with similar stone masses ( $40 \pm 4 \mathrm{mg}$ over $20 \pm 5 \mathrm{~s}$ ) demonstrated a $20 \%$ shorter operating time. However, the muzzle brake tip was able to reduce retropulsion by $50 \%$ and provide minimal tip degradation compared to a bare fiber burnback rate of greater than $500 \mu \mathrm{m} / \mathrm{min}$.

The bare fiber produced an ablation rate of $0.43 \mathrm{mg} / \mathrm{s}$ on a large COM stone when measuring mass loss over time. A sieve to filter smaller fragments was used because surgeons typically would not bother pursuing and ablating fragments smaller than $1.5 \mathrm{~mm}$, since these are already sufficiently small to pass spontaneously from the ureter into the bladder. Using this calculation method, the muzzle brake tips and bare fibers provided ablation rates of 1.6 and $2.0 \mathrm{mg} / \mathrm{s}$, respectively, for smaller stones. However, ablation rate calculations for smaller stones by this method can be misleading as the change in stone mass is minimal to filter through the sieve ( 4 to $1.5 \mathrm{~mm}$ ) and laser operation times are shorter. This slight reduction in stone 
ablation rates with the muzzle brake compared to a bare fiber is possibly due to increased fiber to stone working distance, which was also observed in previous hollow tip TFL laser lithotripsy studies. ${ }^{9}$

Muzzle brake fiber optic tips offer a unique capability to manipulate the vapor bubble generated by high laser pulse energy in aqueous medium. Performance trends were observed based on tip configuration. As expected, based on previous studies, ${ }^{9}$ ablation rates dropped with an increase in fiber recession distance from the tip. This limitation may be eliminated by polishing the inner surface of the steel tip and/or reducing the inner diameter of the tubing, both of which would provide increased energy density at the tip and, therefore, maintain higher ablation rates as the fiber is recessed or degraded.

\subsection{Fiber Tip Degradation}

Fiber tip degradation is caused by the overheating of the fiber tip, resulting in a phase change in the glass, while damage from impact of ejected stone fragments or dust is negligible. ${ }^{8}$ A degradation event occurs when a stone fragment is close to the heated fiber tip during a laser pulse. Larger fiber tips experience less degradation because both energy density and heat generated are lower. While increasing the pulse rate increases the ablation rate, it also generates additional heat and tip degradation. In this study, a lesser tendency for tip degradation was observed if the sheath was located behind the fiber. This may have reduced heating because saline inside the muzzle brake tip cooled the fiber instead of heating the metal. Also, recirculation of debris lodged in the muzzle brake's portholes in certain configurations degraded the fiber tip. A reduction in hole size and/or increased quantity of holes in the muzzle tip may solve this problem. If a major fiber tip degradation event occurred, observed as a large flash, ablation would decrease. Energy tests after degradation recorded a pulse energy of $\sim 25 \mathrm{~mJ}$, below the COM stone ablation threshold for a recessed fiber.

\section{Conclusions}

A muzzle brake fiber tip provided a balance between TFL stone ablation rates, reduced stone retropulsion, and minimal fiber tip degradation. With further development, this fiber tip design could potentially lead to reduced operation times for laser lithotripsy.

\section{Disclosures}

The authors do not have any relevant financial interests in this manuscript.

\section{Acknowledgments}

David Gonzalez was supported by a summer research grant from the Charlotte Research Scholars program at UNC Charlotte. The authors would also like to thank Dr. Richard L. Blackmon for help with the laser setup and imaging in initial exploration of the muzzle brake fiber tips.

\section{References}

1. T. Vos et al., "Global, regional, and national incidence, prevalence, and years lived with disability for 301 acute and chronic diseases and injuries in 188 countries, 1990-2013: a systematic analysis for the Global Burden of Disease Study 2013," Lancet 386(9995) 743-800 (2015).

2. S. Griffin, "Fiber optics for destroying kidney stones," Biophotonics Int. 11(4), 44-47 (2004).
3. A. J. Marks et al., "Holmium:yttrium-aluminum-garnet lithotripsy proximal fiber failures from laser and fiber mismatch," Urology 71(6), 1049-1051 (2008).

4. N. J. Scott, C. M. Cilip, and N. M. Fried, "Thulium fiber laser ablation of urinary stones through small-core optical fibers," IEEE J. Sel. Top. Quantum Electron. 15(2), 435-440 (2009).

5. R. L. Blackmon, P. B. Irby, and N. M. Fried, "Thulium fiber laser lithotripsy using tapered fibers," Lasers Surg. Med. 42(1), 45-50 (2010).

6. R. L. Blackmon, P. B. Irby, and N. M. Fried, "Holmium:YAG $(\lambda=2$, $120 \mathrm{~nm})$ versus thulium fiber $(\lambda=1,908 \mathrm{~nm})$ laser lithotripsy," Lasers Surg. Med. 42(3), 232-236 (2010).

7. R. L. Blackmon, P. B. Irby, and N. M. Fried, "Comparison of holmium:YAG and thulium fiber laser lithotripsy: ablation thresholds, ablation rates, and retropulsion effects," J. Biomed. Opt. 16(7), 071403 (2011).

8. T. C. Hutchens et al., "Detachable fiber optic tips for use in thulium fiber laser lithotripsy," J. Biomed. Opt. 18(3), 038001 (2013).

9. T. C. Hutchens et al., "Hollow steel tips for reducing distal fiber burnback during thulium fiber laser lithotripsy," J. Biomed. Opt. 18(7), 078001 (2013).

10. L. A. Hardy et al., "Thulium fiber laser lithotripsy in an in vitro ureter model," J. Biomed. Opt. 19(12), 128001 (2014).

11. R. L. Blackmon et al., "Thulium fiber laser ablation of kidney stones using a 50- $\mu \mathrm{m}$-core silica optical fiber," Opt. Eng. 54(1), 011004 (2014).

12. L. A. Hardy et al., "Rapid thulium fiber laser lithotripsy at pulse rates up to $500 \mathrm{~Hz}$ using a stone basket," IEEE J. Sel. Top. Quantum Electron. 20(5), 138-141 (2014).

13. C. R. Wilson et al., "A miniaturized, 1.9-French integrated optical fiber and stone basket for use in thulium fiber laser lithotripsy," J. Endourol. 29(10), 1110-1114 (2015).

14. C. R. Wilson et al., "Miniature ball-tip optical fibers for use in thulium fiber laser ablation of kidney stones," J. Biomed. Opt. 21(1), 018003 (2016).

15. C. R. Wilson et al., "Microscopic analysis of laser-induced proximal fiber tip damage during Holmium:YAG and thulium fiber laser lithotripsy," Opt. Eng. 55(4), 046102 (2016).

16. L. A. Hardy et al., "Analysis of thulium fiber laser induced bubble dynamics for ablation of kidney stones," J. Biophotonics (2016).

17. D. Pal et al., "Continuous-wave and quasi-continuous wave thuliumdoped all-fiber laser: implementation on kidney stone fragmentations," Appl. Opt. 55(33), 6151-6155 (2016).

18. V. Zamyatina et al., "Super pulse thulium fiber laser for lithotripsy," Lasers Surg. Med. 48(S27), 10 (2016).

19. K. T. Schomacker et al., "Co: $\mathrm{MgF}_{2}$ laser ablation of tissue: effect of wavelength on ablation threshold and thermal damage," Lasers Surg. Med. 11(2), 141-151 (1991).

20. G. M. Hale and M. R. Querry, "Optical constants of water in the $200 \mathrm{~nm}$ to $200 \mu \mathrm{m}$ wavelength region," Appl. Opt. 12(3), 555-563 (1973).

21. E. D. Jansen et al., "Temperature dependence of the absorptioncoefficient of water for midinfrared laser radiation," Lasers Surg. Med. 14(3), 258-268 (1994).

22. B. I. Lange, T. Brendel, and G. Hüttmann, "Temperature dependence of light absorption in water at holmium and Thulium laser wavelengths," Appl. Opt. 41(27), 5797-5803 (2002).

23. B. E. Knudsen et al., "Performance and safety of Holmium:YAG laser optical fibers," J. Endourol. 19(9), 1092-1097 (2005).

24. A. C. Mues, J. M. Teichmann, and B. E. Knudsen, "Quantification of holmium:yttrium aluminum garnet optical tip degradation," J. Endourol. 23(9), 1425-1428 (2009).

25. J. P. Clarkin, R. J. Timmerman, and J. H. Shannon, "Shaped fiber tips for medical and industrial applications," Proc. SPIE 5317, 70 (2004).

26. D. M. Wilson, "Clinical and laboratory approaches for evaluation of nephrolithiasis," J. Urol. 141(3 Pt 2), 770-774 (1989).

27. M. D. White et al., "Evaluation of retropulsion caused by Holmium: YAG laser with various power settings and fibers," J. Endourol. 12(2), 183-186 (1998).

28. H. Lee et al., "Stone retropulsion during Holmium:YAG Lithotripsy," J. Urol. 169(3), 881-885 (2003).

29. D. S. Finley et al., "Effect of Holmium:YAG laser pulse width on lithotripsy retropulsion in vitro," J. Endourol. 19(8), 1041-1044 (2005). 
30. H. W. Kang et al., "Dependence of calculus retropulsion on pulse duration during Ho:YAG laser lithotripsy," Lasers Surg. Med. 38(8), 762-772 (2006)

31. W. Kamal et al., "Stone retropulsion with Ho:YAG and Tm:YAG lasers: a clinical practice-oriented experimental study," J. Endourol. 30(11), 1145-1149 (2016).

Thomas C. Hutchens is currently a postdoctoral fellow in the Department of Physics and Optical Science at the University of North Carolina at Charlotte.
David A. Gonzalez is currently an undergraduate physics major at the University of North Carolina at Charlotte.

Pierce B. Irby is currently the director of the Kidney Stone Center in the McKay Department of Urology at Carolinas Medical Center.

Nathaniel M. Fried is currently a professor in the Department of Physics and Optical Science at the University of North Carolina at Charlotte and an adjunct faculty member in the McKay Department of Urology at Carolinas Medical Center. His research interests include therapeutic and diagnostic applications of lasers in urology. 